\title{
RADIATION FROM CONDENSED SURFACE OF MAGNETIC NEUTRON STARS
}

\author{
Matthew van Adelsberg, ${ }^{1}$ Dong Lai, ${ }^{1}$ and Alexander Y. Potekhin ${ }^{2,3}$ \\ ${ }^{1}$ Center for Radiophysics and Space Research, Department of Astronomy, Cornell Univer- \\ sity, Ithaca, NY 14853; mvanadel@astro.cornell.edu, dong@astro.cornell.edu ${ }^{2}$ Ioffe Physico- \\ Technical Institute, Politekhnicheskaya 26194021 St.Petersburg, Russia; palex@astro.ioffe.ru, \\ ${ }^{3}$ Isaac Newton Institute of Chile, St. Petersburg Branch, Russia
}

\begin{abstract}
Recent observations show that thermal X-ray spectra of many isolated neutron stars are featureless and in some cases well fit by a blackbody. Such perfect blackbody spectra are puzzling as radiative transport through typical neutron star atmospheres causes noticeable deviation from blackbody. Previous studies have shown that in a strong magnetic field, the outermost layer of the neutron star may be in a condensed solid or liquid form because of the enhanced cohesive energy of the condensed matter. Thus, thermal radiation can emerge directly from the degenerate metallic condensed surface, without going through a gaseous atmosphere. Here we discuss emission properties of condensed $\mathrm{Fe}$ and $\mathrm{H}$ surfaces of magnetic neutron stars in the regimes where such condensation may be possible.
\end{abstract}

Keywords: $\quad$ stars:magnetic fields - stars:neutron - radiation mechanisms:thermal - X-rays:stars

\section{Introduction}

So far about $20 \mathrm{NSs}$ have been detected in thermal emission (for a review see Pavlov \& Zavlin 2003). With the exception of 3-4 sources, the thermal spectra of isolated NSs are featureless and sometimes well fit by a blackbody. For example, deep observations with Chandra and XMM-Newton show that the soft X-ray (0.15-1 keV) spectrum of RX J1856.5-3754 (Walter et al. 1996) can be fit with an almost perfect blackbody at $k T=64 \mathrm{eV}$ (e.g., Drake et al. 2002; Burwitz et al. 2003).

The featureless, and in some cases "perfect" blackbody spectra observed in isolated NSs are puzzling. This is because a NS atmosphere is not a perfect blackbody emitter due to nongrey opacities. Recently, several groups have suggested that the spectrum of RX J1856.5-3754 might be explained if the NS has a condensed surface with no atmosphere above it (Burwitz et al. 2001,Burwitz et al. 2003,Mori \& Ruderman 2003,Turolla et al. 2004). Lai \& Salpeter 
1997 studied the phase diagram of the H surface layer of a NS and showed that for strong magnetic fields, if the star surface temperature is below a critical value, the atmosphere can undergo a phase transition into a condensed state (see also Lai 2001). For $B \geq 10^{14} \mathrm{G}$, this may occur even for temperatures as high as $10^{6} \mathrm{~K}$.

In this paper, motivated by recent observations of dim isolated NSs, we present calculations of the emissivity of condensed Fe or $\mathrm{H}$ surface of magnetic NSs in the regime where we expect condensation might be possible.

\section{Properties of Condensed Surface Layer}

It is well known that strong magnetic fields can qualitatively change the properties of atoms, molecules and condensed matter. For $B \gg B_{0}\left(B_{0}=\right.$ $Z^{2} e^{3} m_{e}^{2} c / \hbar^{3}=2.35 Z^{2} \times 10^{9} \mathrm{G}$, where $Z$ is the nuclear charge number), the electrons in an atom are confined to the ground Landau level, and the atom is elongated, with greatly enhanced binding energy. Covalent bonding between atoms leads to linear molecular chains, and interactions between molecular chains can lead to the formation of three-dimensional condensed matter (see Lai 2001 for a recent review).

For the case of hydrogen, Lai \& Salpeter 1997 showed that in strong magnetic fields, there exists a critical temperature $T_{\text {crit }}$ below which a phase transition from gaseous to condensed state occurs, with $k T_{\text {crit }}$ about $10 \%$ of the cohesive energy of the condensed hydrogen. Thus, $T_{\text {crit }} \sim 8 \times 10^{4}, 5 \times$ $10^{5}, 10^{6} \mathrm{~K}$ for $B=10^{13}, 10^{14}, 5 \times 10^{14} \mathrm{G}$ (Lai 2001).

For heavy elements such as Fe, our current knowledge of the various forms of matter in strong magnetic fields is quite incomplete. Numerical results of Jones 1986, together with approximate scaling relations suggest an upper limit of the cohesive energy (for $Z \geq 10$ ) of $Q_{s} \leq Z^{9 / 5} B_{12}^{2 / 5} \mathrm{eV}$, where $B_{12}=$ $B /\left(10^{12} \mathrm{G}\right)$. Thus for Fe, the critical temperature for phase transition $T_{\text {crit }} \leq$ $0.1 Q_{s} / k \leq 10^{5.5} B_{12}^{2 / 5} \mathrm{~K}$ (Lai 2001).

The emissivity of the condensed surface layer is related to the reflectivity $R$ of the surface through Kirchoff's Law $I_{\nu}^{(e)}=(1-R) B_{\nu}(T)$. As a first approximation, we use the free electron gas model for our calculations. The dielectric tensor for the condensed layer takes the form of Ginzburg 1970, with the collisional damping rates calculated as described by Potekhin 1999. For more details, see van Adelsberg et al. 2004.

\section{Emission from Condensed Surface Layer}

We present results of surface emission for several cases: Figs. 1a and $1 \mathrm{~b}$ show the dimensionless emissivity $J=1-R$ as a function of photon energy $E$ for Fe surface at $10^{13} \mathrm{G}$, and $\mathrm{H}$ surface at $10^{14} \mathrm{G}$, respectively. $T=10^{6} \mathrm{~K}$ 


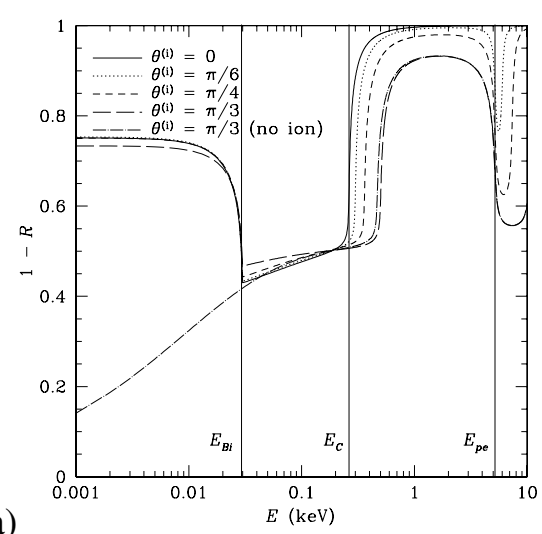

(a)

(b)

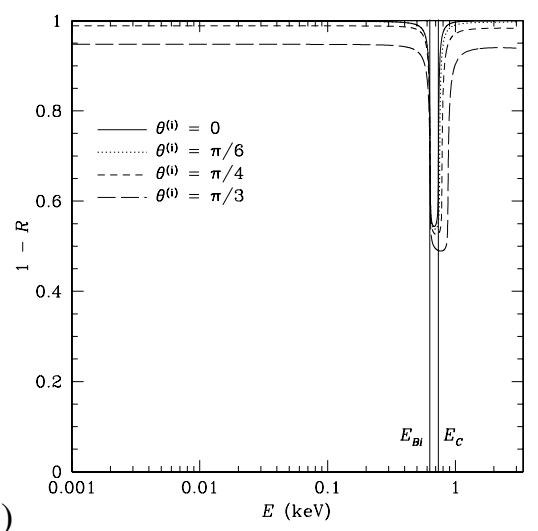

Figure 1. Dimensionless emissivity for Fe surface at $B=10^{13} \mathrm{G}$ (a) and $\mathrm{H}$ surface at $B=$ $10^{14} \mathrm{G}(\mathrm{b})$.

in all our calculations, and the $B$ field is assumed to be normal to the surface (the features in Figs. 1a and $1 \mathrm{~b}$ are representative of our results for more complicated geometries). In both cases, the emissivity is reduced from blackbody at low energies, approaching unity for energies a few times the electron plasma energy $E_{p e}$, and there is feature associated with the ion cyclotron energy $E_{B i}$. For $\mathrm{H}, E_{p e}$ is too high to be of observational interest.

Figure 2 depicts specific flux as a function of photon energy for Fe surface composition at $B=10^{12}, 10^{13} \mathrm{G}$, and $\mathrm{H}$ surface for $B=10^{14} \mathrm{G}$. For the case of $\mathrm{Fe}$, there is a reduced emission (by a factor of 2 or so) around $E_{B i} \leq E \leq$ $E_{c}$ compared to the blackbody at the same temperature. For the $\mathrm{H}$ surface at $B=10^{14} \mathrm{G}$, the flux is close to blackbody at all energies except for a broad feature around $E_{B i}$. Our calculations show that the emission spectrum resembles that of a diluted blackbody, with the reduction factor in the range of $J=0.4-1$ depending on the photon energy (see Figs. $1 \& 2$ ). This would increase the inferred emission radius by a factor of $J^{-1 / 2}$. We note that the results presented in this paper correspond to a local patch of the NS; combining the emission from different surface elements will likely result in smoothing the absorption features of Figs. 1\& 2. Furthermore, we have assumed a perfectly smooth surface. This is valid if the condensed matter is in a liquid state, as is likely to be the case for $\mathrm{H}$ condensate (see van Adelsberg et al. 2004). For Fe, the condensed surface is most likely a solid and we may expect a rough surface. If this is the case, the surface may be much less reflective than the results shown in Figs. $1 \& 2$, and the emission will be closer to the blackbody spectrum. The emission from a condensed NS surface is distinct from atmospheric emission in several aspects: (i) Atmospheric emission generally possesses a hard spectral 


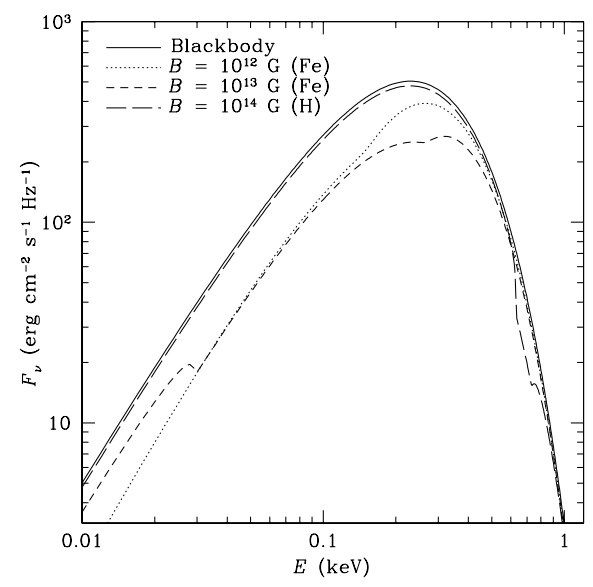

Figure 2. Specific flux for Fe surface at $B=10^{12}, 10^{13} \mathrm{G}$ and $\mathrm{H}$ at $B=10^{14} \mathrm{G}$.

tail whereas the condensed surface emission does not; (ii) The spectrum of a cool NS atmosphere can have both cyclotron and atomic absorption features (iii) The polarization signature of condensed matter emission is qualitatively different from that of atmospheric emission.

\section{References}

Brinkmann, W. 1980, A\&A, 82, 352

Burwitz, V., Zavlin, V.E., Neuhäuser, R., Predehl, P., Trümper, \& J., Brinkman, A.C. 2001, A\&A, 379, L35

Burwitz, V., Haberl, F., Neuhäuser, R., Predehl, P., Trümper, J., \& Zavlin, V.E. 2003, A\&A, 399, 1109

Drake, J., et al. 2002, ApJ, 572, 996

Ginzburg, V.L. 1970, Propagation of Electromagnetic Waves in Plasmas (2d ed.; Oxford: Pergamon Press)

Jones, P.B. 1986, MNRAS, 218, 477

Lai, D. 2001, Review of Modern Physics, 73, 629

Lai, D., \& Salpeter, E.E. 1997, ApJ, 491, 270

Mori, K., \& Ruderman, M. 2003, ApJ, 592, L75

Pavlov, G. G., \& Zavlin, V. E. 2003, in XXI Texas Symposium on Relativistic Astrophysics, ed. R. Bandiera et al. (Singapore: World Scientific), 319

Pons, J.A., Walter, F.M., Lattimer, J.M., Prakash, M., Neuhäuser, R., \& An, P. 2002, ApJ, 564, 981

Potekhin, A.Y. 1999, A\&A, 351, 787

Turolla, R., Zane, S., \& Drake, J.J. 2004, ApJ, 603, 265

van Adelsberg, M., Lai, D., Potekhin, A. 2004, ApJ, submitted

Walter, F., Wolk, S.J., \& Neuhauser, R. 1996, Nature, 379, 233 
PULSARS 